\title{
Early detection of Blunt traumatic brachial artery pseudoaneurysm by point of care ultrasound: case report
}

\author{
Jen-Tang Sun ${ }^{1 *}$, Ming-TseJ Tsai ${ }^{1}$, Chun-Yen Huang ${ }^{1}$, Hong-Wei Chen ${ }^{1}$, Kuang-Chau Tsai ${ }^{1}$, Wan-Ching Lien², \\ Hsiu-po Wang $^{3}$
}

From 10th WINFOCUS World Congress on Ultrasound in Emergency and Critical Care Kuala Lumpur, Malaysia. 16-19 November 2014

\section{Background}

Traumatic pseudoaneurysm(PA) most commonly with penetrating injury, Blunt mechanism is very rare and often delay diagnosis. We present one case of blunt injury induced brachial artery PA diagnosed by point of care ultrasound(US).

\section{Patient and method}

A 61-year-old man with hypertension history, presented with progressive left elbow pain and swelling after blunt injury. He was fell down 1 week ago and direct contusion to his arm. He reported no numbness or weakness. His vital signs were stable except high blood pressure $(188 / 108 \mathrm{mHg})$ Physical examination revealed ecchymosis of his arm (Figure 1) and palpable pulsation over brachial, radial and ulnar artery. Laboratory exam showed elevatingCK(793 IU/L) otherwise normal. X-ray exam revealed normal. US revealed an anechoiclesion over elbow with pulsation.(Figures 2 and 3 ) and some heterogenic lesion over muscle layer, PA and hematoma were considered. CTA of extremity showed PA of brachial artery.(Figures 4 and 5) Patient received endografting and fasciotomy, patient was discharged smoothly after 10 days admission.

\section{Result}

Brachial artery PA can be from 3 etiology:congenital, systemic disease, and trauma. Penetrating injury is most common mechanism.1 Clinical symptoms including redness, induration/ pulsatile mass and pain, Most case of

\footnotetext{
* Correspondence: tangtang05231980@hotmail.com

'Department of Emergency Medicine, Far Eastern Memorial Hospital, New Taipei City, Taiwan

Full list of author information is available at the end of the article
}

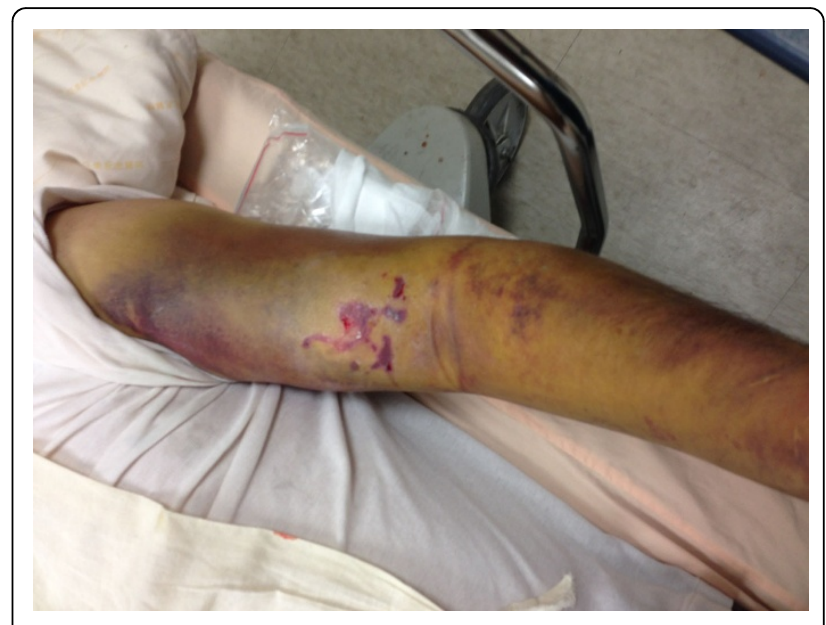

Figure 1

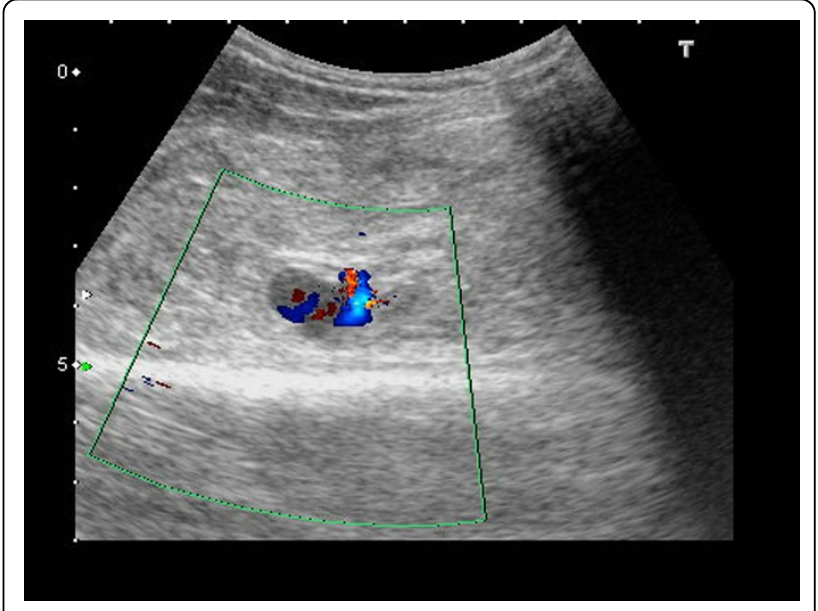

Figure 2

(c) 2015 Sun et al; licensee Springer. This is an Open Access article distributed under the terms of the Creative Commons Attribution License (http://creativecommons.org/licenses/by/4.0), which permits unrestricted use, distribution, and reproduction in any medium provided the original work is properly cited. 


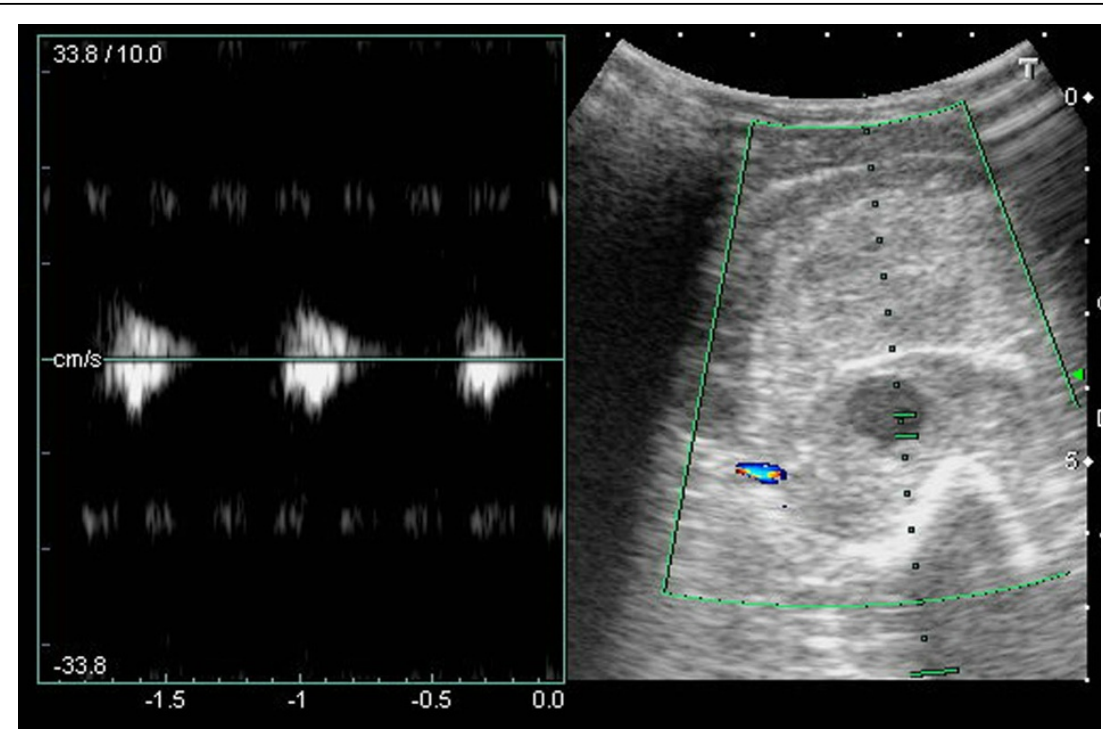

Figure 3

brachial artery PA has delay presentation. In the literature review only 1 case was found for blunt traumatic mechanism not associated without humeral fracture, Inthese two cases were all diagnosed by US. Duplex US has good sensitivity and specificity in postcatheterizationpatients[3] Typical US finding including cystic lesion adjacent to a supplying artery, ying-yang sign in cystic mass, to end fro sign.[4] US also can be used in treatment, like

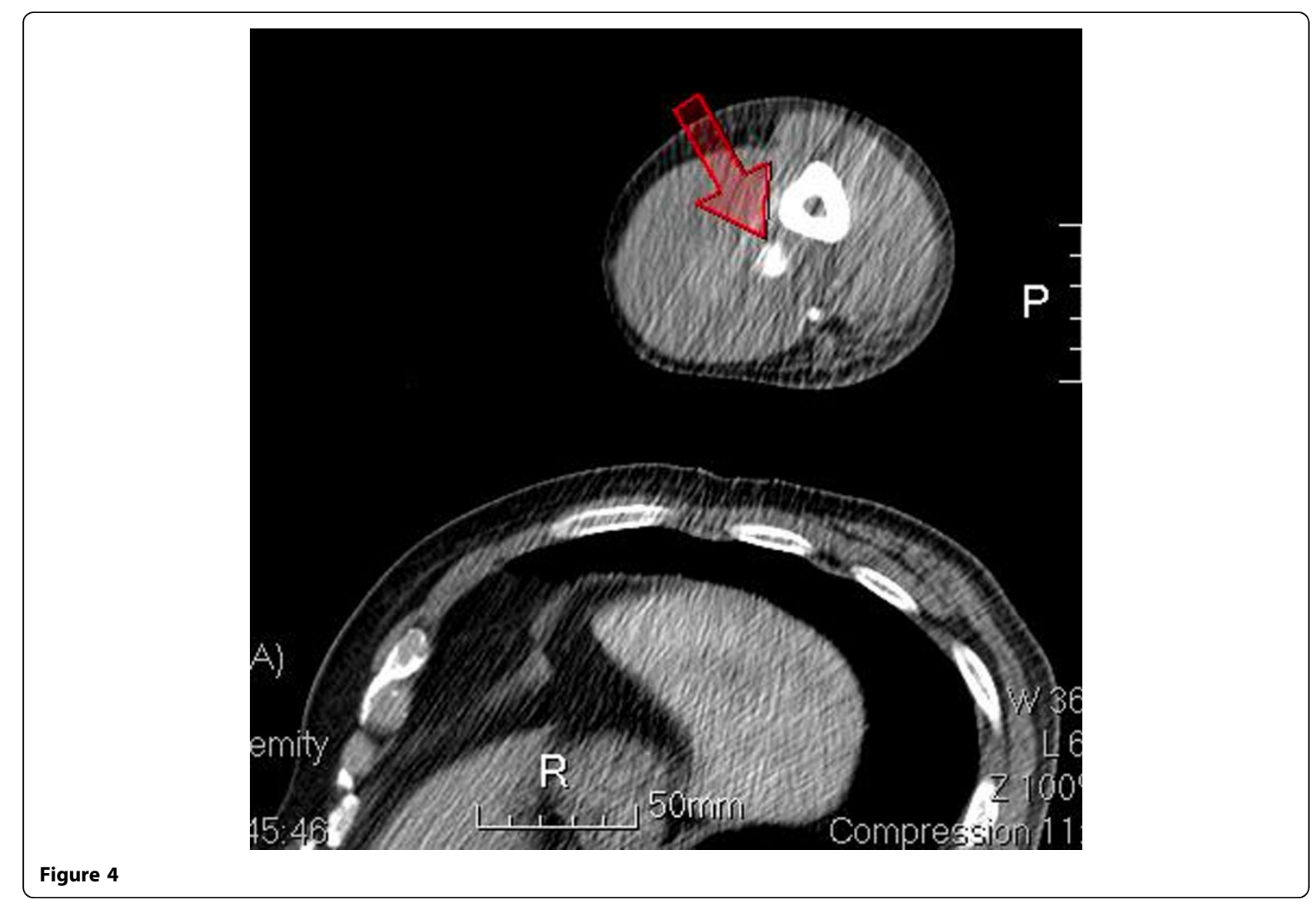




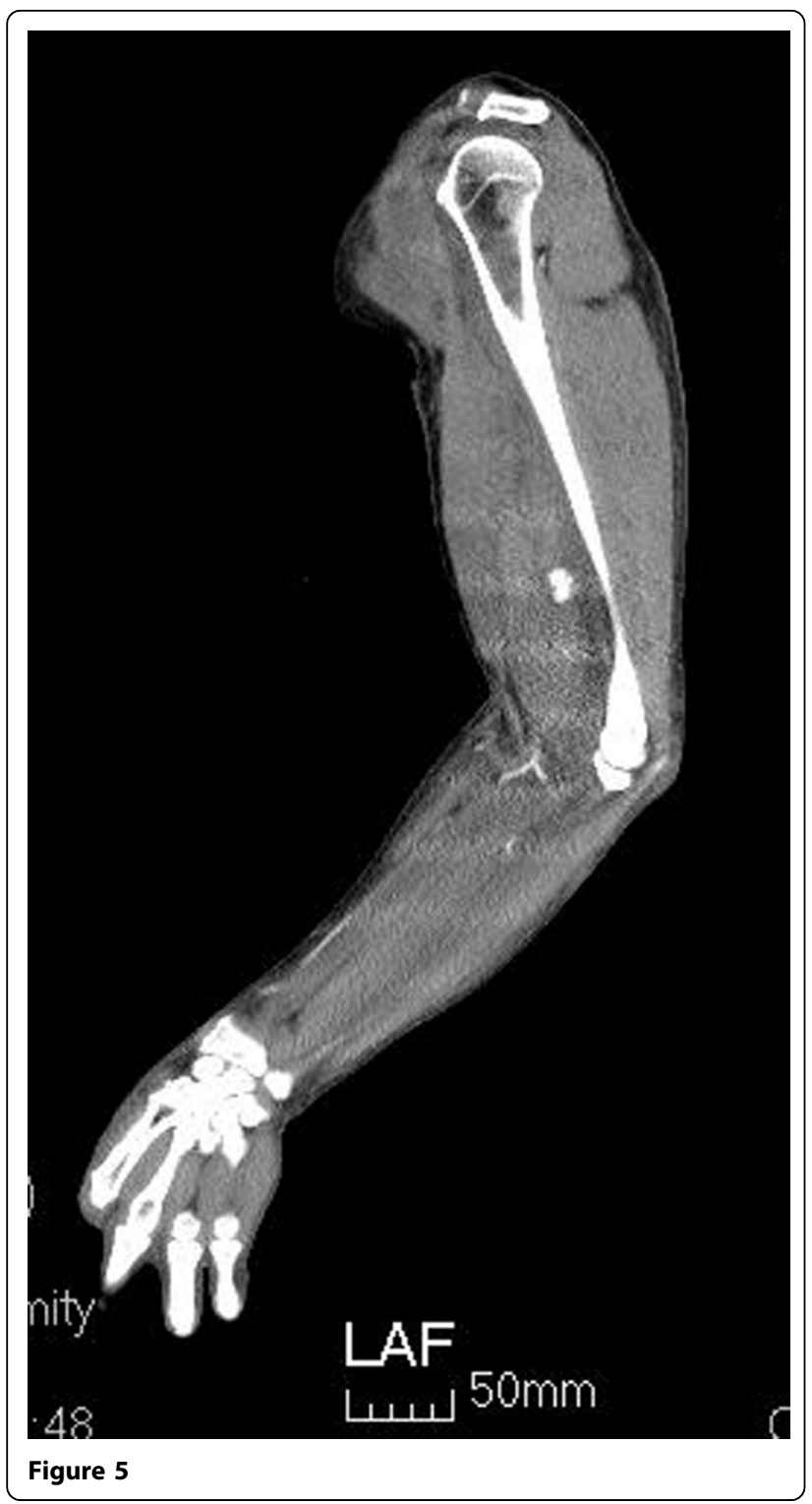

ultrasound guide thrombin injection. Other diagnostic tools including CTA or angiography, but all have radiation.

\section{Conclusion}

Although PA of brachial artery in blunt trauma is rare disease, but should be considered in delay presentation after trauma. Point of care US can properly diagnose the PA in ED setting.

\section{Informed consent}

The study was conducted in accordance with the ethical standards dictated by applicable law. Informed consent was obtained from each owner to enrolment in the study and to the inclusion in this article of information that could potentially lead to their identification.

\section{Authors' details}

'Department of Emergency Medicine, Far Eastern Memorial Hospital, New Taipei City, Taiwan. ${ }^{2}$ Department of Emergency Medicine, National Taiwan University Hospital, Taipei City, Taiwan. ${ }^{3}$ Department of Internal Medicine, National Taiwan University Hospital, Taipei City, Taiwan.

Published: 9 March 2015

\section{References}

1. Lee JY, et al: Delayed rupture of a pseudoaneurysm in the brachial artery of a burn reconstruction patient. World J Emerg Surg 2013, 8(1):21, doi: 10.1186/1749-7922-8-21.

2. Jutte $E H$, et al: Pseudoaneurysm of the brachial artery due to blunt trauma in a child. Cardiovasc Surg 2002, 10(1):52-3.

3. Coughlin BF, et al: Peripheral pseudoaneurysms:evaluation with duplex US. Radiology 1988, 168:339-342.

4. Gullo J, et al: Emergency bedside sonographic diagnosis of subclavian artery pseudoaneurysm with brachial plexopathy after clavicle fracture. Ann Emerg Med 2013, 61(2):204-6.

doi:10.1186/2036-7902-7-S1-A29

Cite this article as: Sun et al: Early detection of Blunt traumatic brachial artery pseudoaneurysm by point of care ultrasound: case report. Critical Ultrasound Journal 2015 7(Suppl 1):A29.

\section{Submit your manuscript to a SpringerOpen ${ }^{\odot}$ journal and benefit from:}

- Convenient online submission

- Rigorous peer review

- Immediate publication on acceptance

- Open access: articles freely available online

- High visibility within the field

- Retaining the copyright to your article 